\title{
Pediatric mandibular osteomyelitis: a probable case from Okhotsk period (5th-13th century AD) northern Japan
}

\author{
Angela R. Lieverse ${ }^{1 *}$, Daisuke KuBO ${ }^{2}$, Rebecca L. Bourgeois ${ }^{3}$, \\ Hirofumi Matsumura ${ }^{4}$, Minoru YonedA ${ }^{5}$, Hajime Ishida ${ }^{6}$ \\ ${ }^{1}$ Archaeology and Anthropology, University of Saskatchewan, Saskatoon, SK, S7N 5B1, Canada \\ ${ }^{2}$ Faculty of Medicine, Hokkaido University, Sapporo 060-8638, Japan \\ ${ }^{3}$ Anthropology, University of Alberta, Edmonton, AB, T6G 2H4, Canada \\ ${ }^{4}$ School of Health Science, Sapporo Medical University, Sapporo 060-8556, Japan \\ ${ }^{5}$ Integrated Biosciences, University of Tokyo, Tokyo 277-8562, Japan \\ ${ }^{6}$ Human Biology and Anatomy, Graduate School of Medicine, University of the Ryukyus, Nishihara 903-0215, Japan
}

Received 28 June 2021; accepted 28 August 2021

\begin{abstract}
This paper discusses a probable case of pediatric mandibular osteomyelitis (OM) from the east Hokkaido Okhotsk (5th-13th century AD) site of Moyoro, Japan. The remains of a young child present an unusual mandibular lesion exhibiting two main features: (1) cortical thickening reflecting periosteal new bone formation, and (2) lytic alveolar destruction with associated antemortem tooth loss. The lesion was examined macroscopically, microscopically, and via computed tomography imaging. A differential diagnosis - considering lesion appearance, location, and the age of the child - is most consistent with OM, while alveolar and dental involvement suggest an odontogenic source such as an infected tooth germ. The infection appears to have been active at the time of death and chronic (i.e. of 4+ weeks) in duration, an interpretation supported by enamel hypoplastic evidence of physiological stress in the preceding 12-18 months. The lesion's unique appearance highlights the diverse manifestation of OM, especially in the jaws and in the absence of modern therapeutic treatment. Despite being considered a relatively common condition among non-adult individuals in the past, surprisingly few cases of pediatric OM have been reported from archaeological contexts. This case, only the second documented on a mandible, contributes to the general paucity of paleopathological literature on OM.
\end{abstract}

Key words: chronic, sclerosing, periostitis, osteitis, Hokkaido

\section{Introduction}

Osteomyelitis (OM) is a nonspecific infection of the bone and marrow. It is usually caused by pyogenic bacteria, most notably Staphylococcus, but other pathogens such as fungi and viruses can also be responsible (Aufderheide and Rodríguez-Martín, 1998; Ortner, 2003; Lewis, 2018; Roberts, 2019). Infection occurs either directly, through an open wound or adjacent soft tissue contamination or, more commonly, through indirect hematogenous dissemination. Because OM specifically involves the marrow, non-adult individuals - whose bones contain high quantities of red marrow-are particularly susceptible (Lewis, 2018). The condition is documented most frequently among children aged 3-12 years, typically manifesting as an acute $(<4$

\footnotetext{
* Correspondence to: Angela R. Lieverse, Department of Archaeology and Anthropology, 55 Campus Drive, University of Saskatchewan, Saskatoon, SK, S7N 5B1, Canada.

E-mail: angela.lieverse@usask.ca

Published online 16 November 2021

in J-STAGE (www.jstage.jst.go.jp) DOI: 10.1537/ase.2108281
}

weeks) infection (Aufderheide and Rodríguez-Martín, 1998; Ortner, 2003; Lewis, 2018; Roberts, 2019). When it spreads hematogenously, as is common among non-adults, then subsequent sepsis may be fatal. In fact, mortality estimates from the pre-antibiotic era were as high as $20-30 \%$ (Bancroft, 1921; Bass, 1928; Amberg and Ghormley, 1934; Lacey and Engel, 1939; Aufderheide and Rodríguez-Martín, 1998).

$\mathrm{OM}$ is a disease with diverse clinical and skeletal manifestations that reflect the combined effects of pathogen virulence, host resistance, and therapeutic treatment. Its study has been plagued by inconsistencies in terminology and diagnostic criteria, hindering both clinical and paleopathological research (Dormans and Drummond, 1994; Aufderheide and Rodríguez-Martín, 1998; Ortner, 2003; Suei et al., 2005; Baltensperger and Eyrich, 2009a, b; Chang et al., 2015; Santos and Suby, 2015; Lewis, 2018; Roberts, 2019). In paleopathology, in particular, pathognomonic lesions such as sequestra, involucra, and cloacae are not always present on skeletal remains. As a result, few archaeological cases of OM have been published, despite the general understanding of the condition as a relatively common and serious health complication in the past (Baltensperger and Eyrich, 2009b; 
Santos and Suby, 2015; Tavares et al., 2017; Lewis, 2018; Roberts, 2019). A thorough documentation of OM prevalence, presentation, and distribution in archaeological and early historical contexts that pre-date the use of antibiotic therapies is paramount to understanding the evolution and long-term impact of the disease (Santos and Suby, 2015). This paper contributes to the paucity of paleopathological literature by presenting a probable case of chronic mandibular OM in a young child from Moyoro, an Okhotsk period site in northern Japan. A differential diagnosis and discussion shed light on the condition's etiology and pathogenesis. To the best of our knowledge, this is only the second case of pediatric mandibular OM documented thus far from the archaeological record.

\section{Materials and Methods}

The Okhotsk were marine-adapted foragers inhabiting Sakhalin, Hokkaido, and the Kuril Islands during the 5th13th centuries AD (Amano, 2003; Hudson, 2004; Naito et al., 2010; Tsutaya et al., 2015; Junno et al., 2021). Their early occupation (5th-6th century) of Sakhalin and north Hokkaido was followed by rapid eastward expansion into east Hokkaido and the Kuril Islands during middle (7th-9th century) period (Yamaura and Ushiro, 1999; Hudson, 2004; Tsutaya et al., 2015). While the Okhotsk relied largely on hunting and fishing, particularly of marine resources, there is also evidence that they consumed domesticated foods including dogs, pigs, barely, and millet (Ishida, 1988; Amano, 2003; Tsutaya et al., 2014; Liepe et al., 2017). However, their eastward migration necessitated a subsistence shift in response to changing winter sea ice conditions: the east Hokkaido Okhotsk did not keep pigs and relied heavily on marine mammals (Yamaura, 1998; Yamaura and Ushiro, 1999; Hudson, 2004; Tsutaya et al., 2014). After remaining distinct for centuries, the Okhotsk were replaced by/assimilated with Satsumon groups, who had Jomon ancestry, at the end of the 10th century AD in north Hokkaido and during the 12th century AD in east Hokkaido (Amano, 2003; Hudson, 2004; Sato et al., 2007, 2009; Tsutaya et al., 2015). The site of Moyoro (Figure 1) is an east Hokkaido Okhotsk shell midden from which $\sim 250$ individuals were excavated between 1926 and 2011 (Kodama, 1948; Komai, 1964; Kiyono, 1969; Abashiri City Board of Education, 2014). Most human remains recovered date between 500 and 900 cal. AD (with application of the Marine13 calibration curve), consistent with the middle Okhotsk period of rapid eastward expansion (Tsutaya et al., 2014, 2015). While several rich Moyoro burials have been noted (Hudson, 2004), there is no information regarding mortuary context for many interments, including the individual discussed here.

Moyoro 165, the subject of this paper, represents the incomplete skeleton (Figure 2) of a young child. The remains are directly dated to $1871 \pm 32 \mathrm{BP}$, or 736-977 cal. AD $(68.3 \%, \pm 1$ SD), with the application of the IntCal20 and Marine 20 calibration curves and assuming 90\% contribution from marine proteins and a local reservoir correction of $+248 \pm 99$ years (based on eight dates from Kunashiri, Shikotan, and Sakhalin; Kuzmin et al., 2001; Yoneda et al., 2007), as calculated with the 14CHRONE Marine20 Reser-

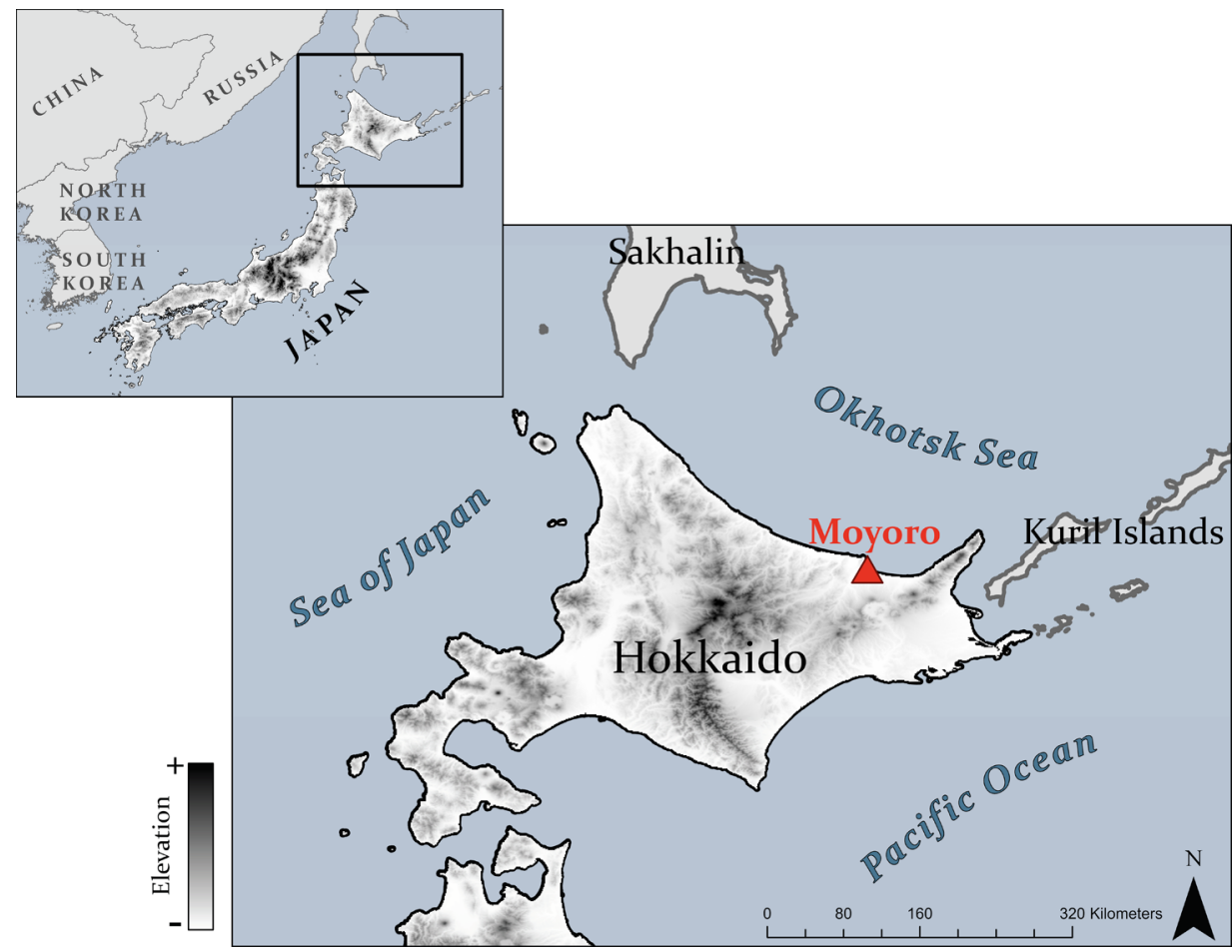

Figure 1. Map of Hokkaido, Japan, on a digital elevation model backdrop. Elevations are depicted on a gradient scale from low (light) to high (dark). The Moyoro site is indicated in red. Data were obtained and are freely available from DIVA-GIS and created using ArcGIS Pro. Image by W. Wadsworth, University of Alberta. Color version available in the online PDF. 


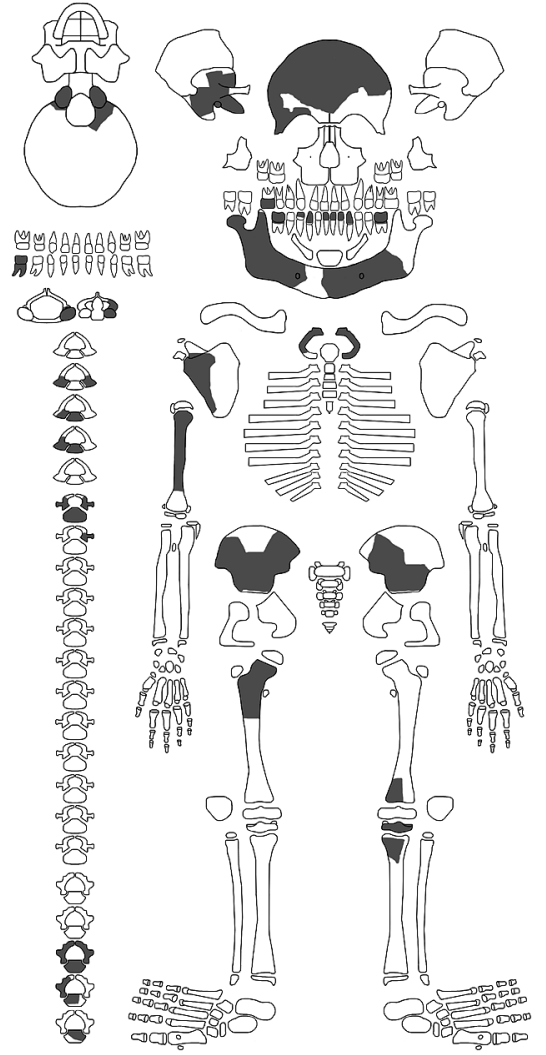

Figure 2. Diagrammatic skeletal/dental inventory for Moyoro 165. Elements/fragments present (recovered postmortem) are shaded in gray. Image by D. Kubo. Template from Roksandic (2003). voir Database. The lesion of interest affects the mandible, which is represented by two fragments: (1) most of the left mandibular body and a small part of the right, and (2) the posterior aspect of the right mandibular body and ramus (Figure 3). An inventory and developmental summary of the mixed mandibular dentition are presented in Table 1, indicating a dental age of 3.5-5.5 years (Ubelaker, 1979; Schaefer et al., 2009; AlQahtani et al., 2010). Of particular note - and directly relevant to the lesion (see below) - is the antemortem loss of both left deciduous molars and the developing replacement permanent premolars. The cranial and postcranial remains are fragmented and incomplete, with occipital and vertebral fusion indicating an age consistent with dental development (Schaefer et al., 2009). Slight porosity was observed on the right orbital roof, and two linear enamel hypoplastic defects were noted on the cervical crowns of each developing permanent mandibular canine (Figure S1), suggesting stress events during the 12-18 months before death (Schaefer et al., 2009; AlQahtani et al., 2010). No other pathological changes were documented, although skeletal preservation is admittedly poor.

\section{Results}

The lesion itself encompasses most of the left mandibular body and exhibits two main features (Figure 3). The first is cortical thickening along the length of the body, most pronounced between the canine and first permanent molar, reflecting periosteal new bone formation on the lateral (buccal), inferior, and medial (lingual) surfaces. The cortex in this area, especially on the lateral surface, is distinctly swol-

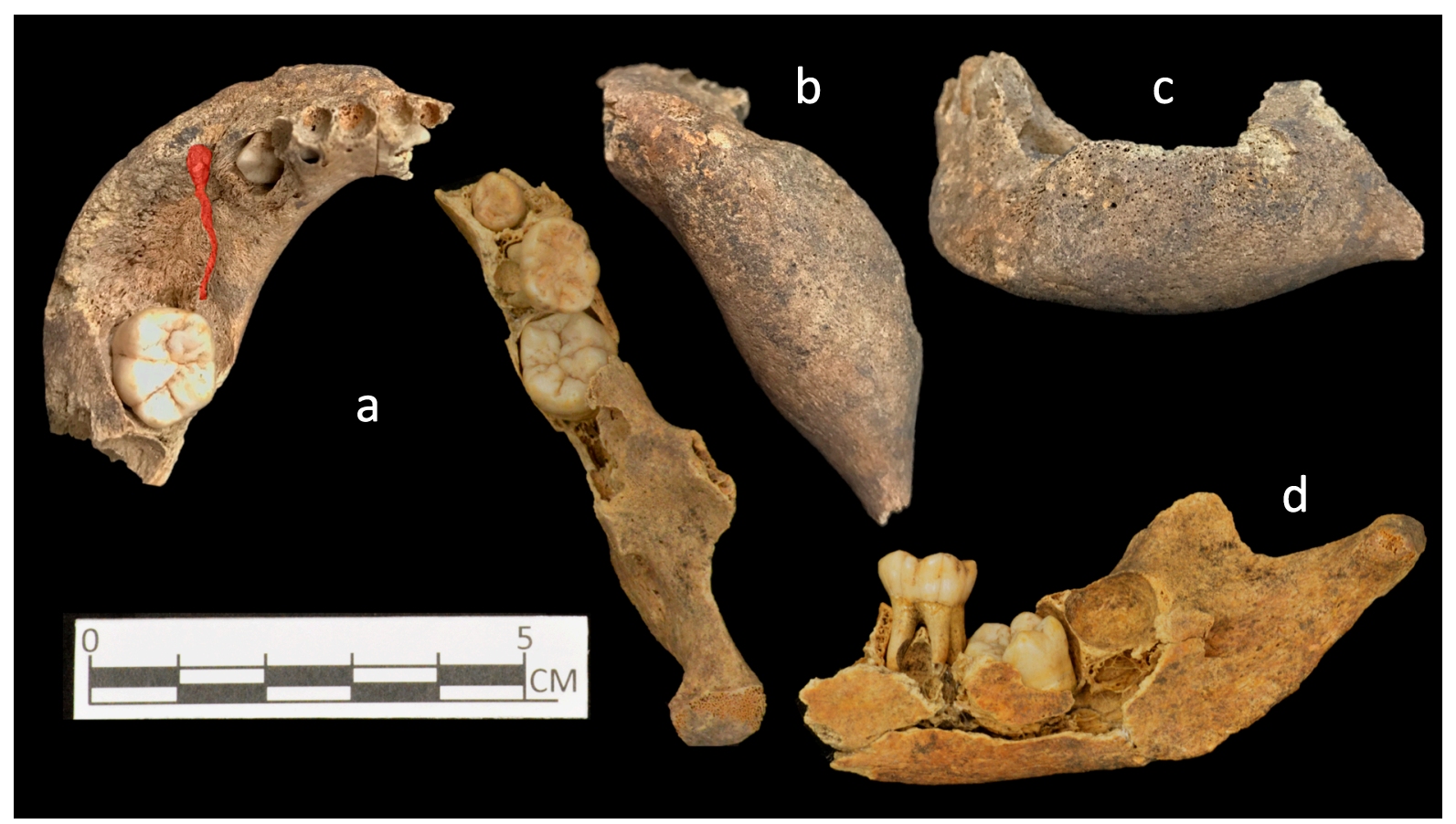

Figure 3. Moyoro 165: (a) occlusal view of pathological left mandibular body and non-pathological right mandibular body, both with recovered teeth in situ and the course of the left mandibular canal shaded in red; (b) pathological left mandibular body, inferior view with lateral (buccal) side on the right; (c) pathological left mandibular body, lateral view; (d) non-pathological right side of mandible, medial (lingual) view indicating dental development. Images by A. Lieverse and D. Kubo. Color version available in the online PDF. 
Left mandibular dentition

Table 1. Moyoro 165 Mandibular Dental Inventory* and Development

\begin{tabular}{|c|c|c|c|}
\hline \multicolumn{2}{|c|}{ Left mandibular dentition } & \multicolumn{2}{|c|}{ Right mandibular dentition } \\
\hline \multicolumn{2}{|c|}{ Deciduous teeth } & \multicolumn{2}{|c|}{ Deciduous teeth } \\
\hline 71 & absent postmortem & 81 & absent postmortem \\
\hline 72 & absent postmortem & 82 & absent postmortem \\
\hline 73 & absent postmortem & 83 & absent postmortem \\
\hline 74 & absent antemortem & 84 & absent postmortem \\
\hline 75 & absent antemortem & 85 & present in socket (tooth fully formed) \\
\hline \multicolumn{2}{|c|}{ Permanent teeth } & \multicolumn{2}{|c|}{ Permanent teeth } \\
\hline 31 & present in crypt (crown $3 / 4+$ formed), visible via CT & 41 & present in crypt (crown complete, root initiated) \\
\hline 32 & present in crypt (crown $3 / 4+$ formed), visible via CT & 42 & absent postmortem \\
\hline 33 & present in crypt (crown $3 / 4$ complete) & 43 & present without crypt (crown $3 / 4$ complete) \\
\hline 34 & absent antemortem & 44 & present in crypt (crown incomplete) \\
\hline 35 & absent antemortem & 45 & absent postmortem \\
\hline 36 & present in crypt (crown complete, root initiated) & 46 & present in crypt (crown complete, root initiated) \\
\hline 37 & absent postmortem & 47 & absent postmortem \\
\hline
\end{tabular}

* Identified using FDI notation (Fédération Dentaire Internationale, 1971); bold font indicates teeth present postmortem

len and slightly roughened, with increasing porosity toward the superolateral margin (Figure 3b, c). The left mandibular body measures $21.8 \mathrm{~mm}$ mediolaterally at its greatest extent (mesial to the first permanent molar), while the right measures $12.5 \mathrm{~mm}$ at the same location. The second lesion feature is a large $(33.2 \mathrm{~mm} \times 21.4 \mathrm{~mm})$ lytic defect of the left alveolar bone between the canine and first permanent molar (Figure 3a). This lesion completely obliterated the sockets of both deciduous molars, the crypts of both developing permanent premolars, and most of the mandibular canal anterior (mesial) to the first permanent molar. The lateral and medial margins of the defect are elevated (superiorly) relative to its interior and are distinctly porous, particularly on the lateral side (Figure 3c). The entire base of the lytic lesion consists of undulating trabecular bone, with the course of the mandibular canal being vaguely distinguishable (Figure $3 a$ ). The base of the defect was further examined via a handheld digital microscope (Figure 4).

Clinical computed tomography (CT) scanning was conducted with a NAOMi-CT 3D-M (RF Co.) at the Sapporo Medical University by H. Matsumura. The images were reconstructed with isotropic $0.071-\mathrm{mm}$ voxels. CT imaging confirms that the expansion of the left mandibular body reflects periosteal new bone formation on the lateral, inferior,

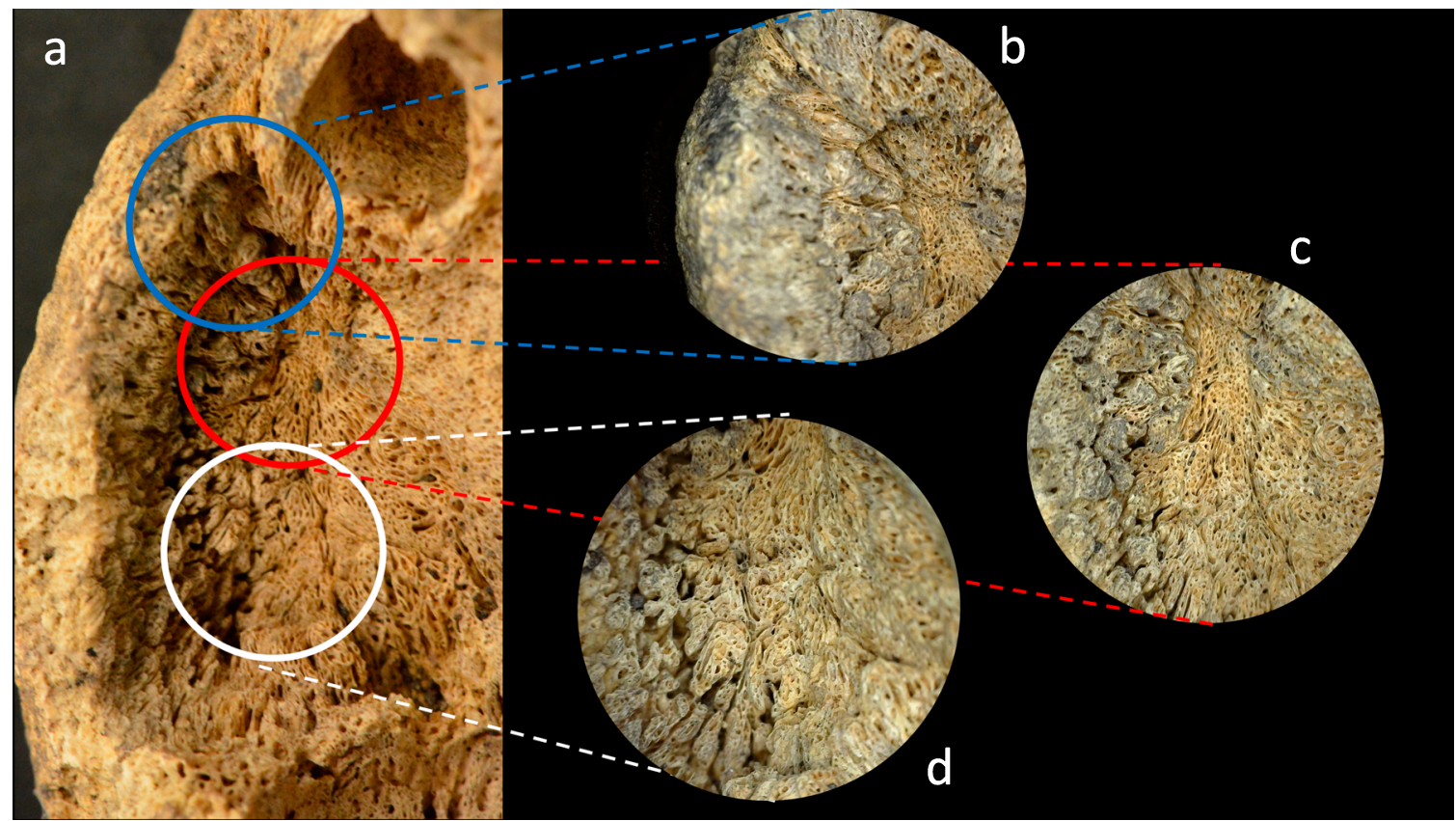

Figure 4. Moyoro 165: (a) occlusal view of the lytic alveolar lesion on the left mandibular body; (b-d) close-up views of the base of lytic lesion, $\sim 30 \times$ magnification (taken with a Dino Lite digital microscope). Images by A. Lieverse and D. Kubo. Color version available in the online PDF. 


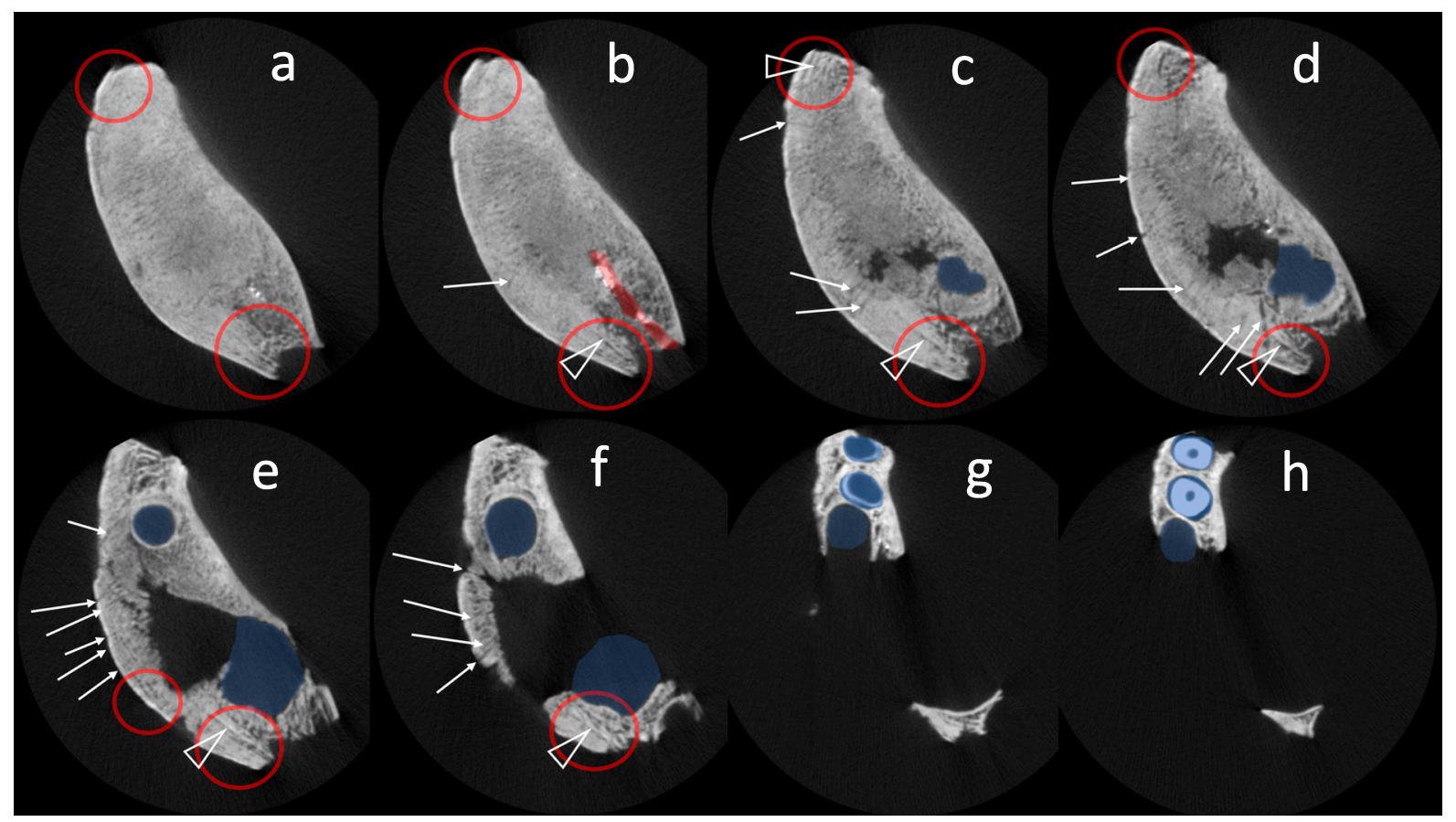

Figure 5. Moyoro 165: series of clinical CT images taken of the left mandibular body in the transverse plane from the inferior (a) through superior/occlusal (h). The mesial portion of the mandibular body is oriented toward the top of the images and the lateral side is on the left. Blue shaded areas are dental crypts/developing teeth, while the red shaded area (b) is the intact mandibular canal. Open triangles (b-f) identify remnants of original cortical bone and red circles $(\mathrm{a}-\mathrm{f})$ denote lamellated areas of periosteal new bone formation. White arrows $(\mathrm{b}-\mathrm{f})$ indicate some of the small canals extending outward from the internal mandibular body. Color version available in the online PDF.

and medial surfaces (Figure 5, Figure 6). Much of this bone is at least partially remodeled and integrated with the original cortex, but areas of lamellated ('onion-skin') deposits are visible on the lateral mandibular body (Figure 5a-f, Figure $6 \mathrm{a}, \mathrm{c}-\mathrm{d}$ ). The lytic alveolar lesion appears to interrupt the periosteal new bone in the area of the deciduous molars and permanent premolars (Figure 5c-e, Figure 6c). There is no evidence of these teeth or their dental crypts, but the crowns and partial roots of the developing left permanent incisors are visible in the anterior portion of the mandible (Figure $5 \mathrm{~g}-\mathrm{h}$ ). The mandibular canal appears to be intact posterior (distal) to the alveolar defect (Figure 5b, Figure 6a, c). The CT scans also reveal (1) dense trabecular bone in the interior mandibular body, especially inferior to the alveolar lesion (Figure 5a-c, Figure 6a, b); (2) undulating trabeculae comprising the base and margins of the lytic lesion (Figure $5 \mathrm{e}-\mathrm{f}$, Figure $6 \mathrm{~b}-\mathrm{d}$ ); and (3) numerous small canals extending outward from the internal mandibular body, particularly from the area of the lytic lesion (Figure 5b-f, Figure 6). Most of these canals, especially on the more inferior portion of the bone, do not perforate the external cortex. Those that do appear to correlate with the porosity observable on the lateral surface (Figure 3b, c). No evidence of trauma (e.g., fractures) or abscess formation (e.g., cloacae) was observed on the mandible macroscopically, microscopically, or via CT imaging. No destructive analyses were permitted for these remains.

\section{Differential Diagnosis}

The two distinct lesion features on the Moyoro 165 mandible, one osteoblastic and the other osteolytic, complicate differential diagnosis somewhat. Pronounced periosteal new bone formation on the mandibular body may reflect a number of different pathological conditions in a child, including hypervitaminosis A, infantile cortical hyperostosis, malignant neoplasia, treponemal disease, fibro-osseous lesions, Langerhans cell histiocytosis, and osteomyelitis (Belli et al., 2002; Fukuda et al., 2017; Lewis, 2018). Lytic alveolar destruction, on the other hand, can result from some of the same conditions - malignant neoplasia, Langerhans cell histiocytosis, and osteomyelitis - as well as tuberculosis (Can et al., 2005; Baltensperger and Eyrich, 2009a; Lewis, 2018; Hwang et al., 2019).

Hypervitaminosis A, or vitamin A toxicity, reduces bone formation and enhances its resorption (Binkley and Kreuger, 2000). The condition has been associated with a proliferation of coarse periosteal bone on the skull and long bones and, in non-adults, delayed ossification (Naz and Edwards, 1952; Woodard et al., 1961; Walker et al., 1982; Aufderheide and Rodríguez-Martín, 1998). In the case of the Moyoro 165 , bone formation appears to be limited to the mandible and is distinct in its lamellated appearance. This, and the singular lytic alveolar defect, is inconsistent with hypervitaminosis A. Infantile cortical hyperostosis (ICH) has a poorly understood etiology that may include viral infection, trauma, arterial morphology, and even hypervitaminosis A (Lewis, 2018). It is characterized by widespread formation of peri- 

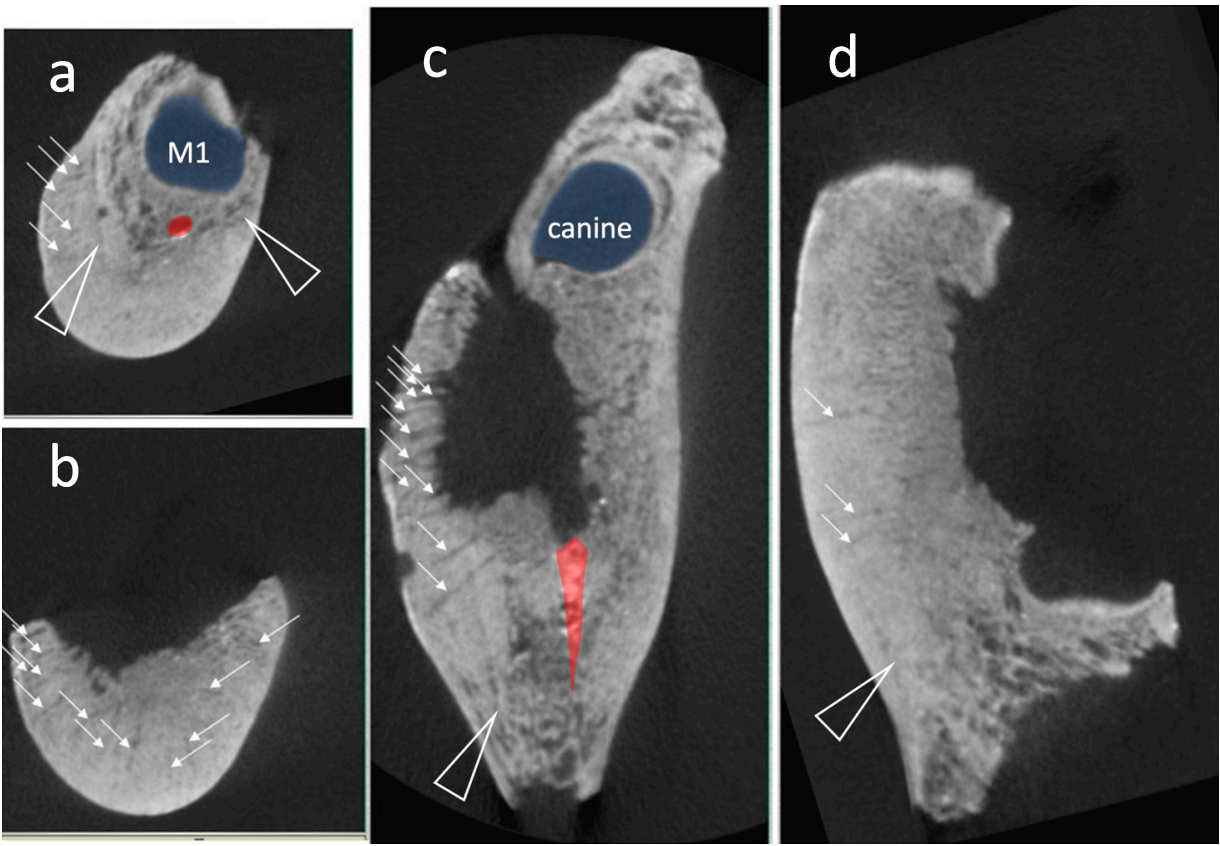

Figure 6. Moyoro 165: clinical CT images taken of the left mandibular body: (a) coronal plane through the developing first permanent molar crypt; (b) coronal plane through the lytic alveolar lesion; (c) transverse plane through the lytic lesion and inferior to the developing first molar crypt; (d), sagittal plane through the alveolar lesion. Blue shaded areas (a, c) are dental crypts and red shaded areas (a, c) identify the intact mandibular canal. Open triangles $(\mathrm{a}, \mathrm{c}-\mathrm{d})$ indicate remnants of original cortical bone, while white arrows denote some of the small canals extending outward from the internal mandibular body. Color version available in the online PDF.

osteal new bone and cortical thickening on multiple elements, most often the skull, long bones, and ribs (KamounGoldrat and le Merrer, 2008; Lewis, 2018). On the mandible, the condition is more common on the ramus and angle, rather than the body (Karjodkar, 2009). The diffuse lesion distribution of ICH and its occurrence on young infants, typically manifesting before five months of age (Caffey, 1945; Kamoun-Goldrat and le Merrer, 2008), make it an unlikely diagnosis in this case.

Malignant neoplastic conditions affecting young children, such as leukemia, Ewing's sarcoma, and metastatic neuroblastoma, may also account for the periosteal bone forming lesion and/or lytic alveolar defect on the Moyoro 165 mandible. Leukemia, acute leukemia in particular, is the most common malignant condition affecting children (Kobayashi et al., 2005), with bony involvement present in at least 50\% of cases (Sugihara et al., 1989). Skeletal changes are typically multifocal and characterized by widespread periosteal bone deposition and osteolysis. In the jaws, lytic lesions can result in trabecular loss, thinning dental crypts, and tooth displacement (Sugihara et al., 1989; Kobayashi et al., 2005; Lewis, 2018). In this case, the localized and partially lamellated periosteal bone formation and single alveolar defect are inconsistent with leukemia. Furthermore, some of the most characteristic features of childhood leukemia, enlarged vascular foramina and exaggerated porosity and sulci on the metaphyseal cortices (Grauer, 2019), are absent on this individual. Ewing's sarcoma and neuroblastoma are two other childhood malignancies that also cause osteoblastic lesions, including 'onion skin', sunburst, or hair-on-end reactions. However, these are typically aggressive bony expansions accompanied by osteolysis (e.g., mottling) visible on radiographs (Campanacci et al., 1990; Belli et al., 2002; White and Pharoah, 2009; Akgül et al., 2018; Botía González et al., 2018; Lewis, 2018), which are absent here. The localized and partially remodeled bone formation on Moyoro 165 does not support a malignant diagnosis.

Fibro-osseous lesions that may account for mandibular expansion in children include fibrous dysplasia and cherubism. Both are benign conditions reflecting an overproduction of fibrous tissue within bone (Papadaki et al., 2012; Lewis, 2018; Marques, 2019). Fibrous dysplasia can affect one or several bones, the latter typically asymmetrically, while cherubism is usually symmetrical and limited to the mandible and maxillae. Radiographically, both conditions are distinguishable by their 'ground glass' appearance (Kambadakone et al., 2008; Craig and Craig, 2013; Willmon et al., 2013; Lewis, 2018; Marques, 2019), which is absent in this case (Figure 5, Figure 6). Fibro-osseous lesions are therefore inconsistent with the osteoblastic lesion on Moyoro 165.

Infectious diseases-specifically treponematosis (one of four conditions caused by Treponema pallidum) and tuberculosis (caused by Mycobacterium, usually M. tuberculosis) - are also possible diagnoses. Treponemal infections potentially affecting the jaws of young children are largely limited to congenital syphilis (Roberts and Buikstra, 2019). Associated skeletal changes include profuse periosteal bone formation, gummatous lesions on the skull, and 'saddle nose' facial deformities. Pathognomonic dental changes, such as hypoplastic teeth, mulberry molars, and Hutchinson's incisors, are most common, occurring in up to $45 \%$ of infected individuals (Lewis, 2018; Roberts and Buikstra, 
2019). In the case of Moyoro 165, the solitary and localized nature of the bone deposition and the typical morphology of all ten (nine mandibular and one maxillary) observable teeth make treponematosis unlikely. Tuberculosis (TB), while rarely affecting the mandible, does so more commonly in infants and children than in adults (Aufderheide and Rodríguez-Martín, 1998; Lewis, 2018; Roberts and Buikstra, 2019). TB lesions on non-adult skeletal remains are usually multifocal, rather than solitary, and predominately osteolytic. On some elements, such as the mandible, lesions may mimic those of Langerhans cell histiocytosis (Lewis, 2018; Roberts and Buikstra, 2019), making differential diagnoses difficult. In this case, the solitary nature of the lytic alveolar lesion, lack of destructive foci elsewhere on the skeleton, and apparent absence of the disease from Hokkaido until the mid-1600s (Kondo and Aono, 2016) all argue against a diagnosis of TB.

Langerhans cell histiocytosis ( $\mathrm{LCH})$ is a reticuloendothelial disease in which an overproduction of histocytes called Langerhans cells causes destructive granulomatous lesions in skeletal tissues and other organs (Willman et al., 1994; Lewis, 2018; Grauer, 2019). Its peak occurrence is among children under the age of 10 , but its presentation can vary widely depending on distribution, severity of skeletal involvement, and age of onset (Lewis, 2018; Grauer, 2019). In the paleopathological literature, LCH is generally classified into one of three diseases (Grauer, 2019). Eosinophilic granuloma is the most common and least severe form of $\mathrm{LCH}$, characterized by solitary lytic lesions that often involve the cranial vault or facial skeleton. In the mandible, where the body or angle is typically affected, alveolar destruction can cause radiographic 'floating' teeth, tooth loss, and disrupted dental development (Watzke et al, 2000; Can et al., 2005; Mitomi et al., 2005; Lewis, 2018; Hwang et al., 2019; Grauer, 2019). Hand-Schüller-Christian Disease and Letterer-Siwe Disease are more severe versions of $\mathrm{LCH}$, together comprising about $30 \%$ of cases. They are characterized by multifocal, coalesced lesions and diffuse skeletal and multi-organ involvement, respectively (Lewis, 2018; Grauer, 2019), none of which appear to be present in this case. LCH lesions can also have sclerotic borders and/or be associated with periosteal new bone formation (Grauer, 2019). However, these are uncommon in children with eosinophilic granuloma and, when present, are usually discontinuous (Yu et al., 1995). The solitary alveolar defect and tooth/crypt loss of Moyoro 165 is consistent with eosinophilic granuloma and, while the adjacent periosteal new bone formation is atypical for the condition, it is not inconsistent. Therefore, $\mathrm{LCH}$, specifically eosinophilic granuloma, is a possible diagnosis for this case.

$\mathrm{OM}$, a bone and marrow infection most commonly affecting children, can be difficult to diagnose from archaeological human remains (Tavares et al., 2017; Lewis, 2018; Roberts, 2019). The condition may present with diverse and even nonspecific skeletal lesions reflecting variation in its manifestation (e.g., acute/subacute versus chronic, or suppurative versus non-suppurative) or infection source (Aufderheide and Rodríguez-Martín, 1998; Ortner, 2003; Suei et al., 2005; Baltensperger and Eyrich, 2009a, b; Lewis, 2018; Roberts, 2019). For example, OM stemming from open wounds or adjacent soft tissue contamination is often localized and restricted to the cortex and periosteum, without clear involvement of the medullary cavity (Ortner, 2003; Roberts, 2019). In fact, characteristic lesions reflecting tissue necrosis (sequestra), periosteal elevation (involucra), and pus drainage (cloacae) are frequently absent, even in cases of acute suppurative infection. In children, this can reflect the ability of exudate to seep through porous immature bone without increasing vascular pressure (Lewis, 2018). Pediatric OM is usually hematogenous in origin, most often initiating in the metaphysis of a single long bone, especially the tibia or femur; it is rare in the skull and jaws (Aufderheide and Rodríguez-Martín, 1998; Ortner, 2003; Lewis, 2018; Roberts, 2019).

$\mathrm{OM}$ of the jaws is a unique condition that continues to present diagnostic challenges, even for modern clinicians (Suei et al., 2005; Baltensperger and Eyrich, 2009b). Like OM elsewhere in the body, hematogenous spread is a known route of infection, especially in the absence of modern therapeutic treatment. However, direct extension from an odontogenic focus is frequently implicated and today accounts for most cases of jaw OM. Among infants and young children, such as Moyoro 165, developing tooth germs represent an additional source of (hematogenous or direct muscosal) infection (Ortner, 2003; Baltensperger and Eyrich, 2009a; Roberts, 2019). Because of its architectural similarities to long bones (i.e., thick cortex and abundant medullary tissue) and more restricted blood supply, the mandible is ten times as likely to develop OM than is the maxilla in individuals over the age of two years (Baranoff, 1934; Ortner, 2003; Baltensperger and Eyrich, 2009a; Roberts, 2019). The mandibular body is most often affected, followed by the symphysis and angle. While mandibular OM can present with pathognomonic lesions, it may also be clinically silent and/ or non-suppurative, characterized by localized nonspecific lesions such as diffuse sclerosis, cortical thickening, and little or no osteolysis (Aufderheide and Rodríguez-Martín, 1998; Ortner, 2003; Baltensperger and Eyrich, 2009a; Lewis, 2018; Roberts, 2019). Furthermore, OM stemming from tooth germ infection can result in the necrosis and extrusion of dental tissues, with subsequent tooth loss and developmental facial deformities (Natvig and Dingman, 1957; Ortner, 2003; Baltensperger and Eyrich, 2009a; Roberts, 2019). Both lesion features of the Moyoro 165 mandible are consistent with a diagnosis of $\mathrm{OM}$, making $\mathrm{OM}$ a more likely diagnosis than LCH in this case.

\section{Discussion}

In paleopathology, OM diagnosis is limited by lesion specificity, with etiology generally focusing on the route of infection rather than pathogen identification. Unfortunately, establishing the cause of $\mathrm{OM}$ from archaeological human remains is not usually possible, except in rare cases of compound fractures (Santos and Suby, 2015; Lewis, 2018). OM of the jaws is distinct in this regard, as its etiology is most often linked to odontogenic sources (Oulis et al., 2000; Belli et al., 2002; Ortner, 2003; Suei et al., 2005; Baltensperger and Eyrich, 2009a; Khudaverdyan, 2011; Waters-Rist, 2012; Chang et al., 2015; Fukuda et al., 2017; Roberts, 2019). 
Direct extension from an odontogenic infection is implicated for Moyoro 165 by the clear evidence of alveolar involvement and tooth loss, and by the localized nature of the lesion. Because developing and erupting teeth are particularly susceptible to infection (Huang, 2009), the Moyoro child's young age is compatible with $\mathrm{OM}$ development secondary to an infected tooth germ (e.g., tooth 34 and/or tooth 35). However, a periapical infection from caries or direct trauma, or a periodontal infection associated with a recently erupted tooth (e.g., tooth 74 and/or 75), are also possible etiological mechanisms (Oulis et al., 2000; Belli et al., 2002; Chang et al., 2015; Fukuda et al., 2017).

The pathogenesis of $\mathrm{OM}$ reflects the complex interplay between infection virulence and the effectiveness of the host's immune response (Aufderheide and RodríguezMartín, 1998; Baltensperger and Eyrich, 2009a). Acute OM $(<4$ weeks) that is eradicated quickly indicates a strong response relative to pathogen number and/or virulence, whereas acute OM that is fatal, occurring in $20-30 \%$ of pre-antibiotic era cases (Bancroft, 1921; Bass, 1928; Amberg and Ghormley, 1934; Lacey and Engel, 1939; Aufderheide and Rodríguez-Martín 1998), suggests the opposite. Chronic OM (4+ weeks) usually reflects the host's failure to resolve the acute stage of the disease, with the infection invading deep into the cortical bone and medullary cavity. Its manifestation, especially in the jaws, can be quite varied (e.g., Oulis et al., 2000; Belli et al., 2002; Ortner, 2003; Suei et al., 2005; Baltensperger and Eyrich, 2009a; Khudaverdyan, 2011; Waters-Rist, 2012; Chang et al., 2015; Fukuda et al., 2017). In the case of Moyoro 165, the chronic nature of the condition is demonstrated by lamellated periosteal bone formation, partial remodeling, and dense trabeculae comprising much of the mandible's interior. Furthermore, the infection appears to have been active at the time of death, as the porous and slightly roughened cortical bone is inconsistent with a partially or completely healed lesion. Thus, infection likely persisted for at least several months, and possibly longer than a year, prior to death. While local immunological and microbial factors would have played a considerable role in the condition's pathogenesis (Baltensperger and Eyrich, 2009a), at this point it is not possible to link these to aspects of Okhotsk cultural practice or diet.

Skeletal lesions pathognomonic of OM reflect the combined effects of vascular obstruction (tissue necrosis) and pus formation (periosteal elevation and drainage cloacae). The anatomic structure of the mandible makes it particularly vulnerable to infection, and thus these changes. For example, most of the of the blood supply to the mandibular body is delivered via the small inferior alveolar artery, passing through the mandibular canal (Castelli, 1963; Baltensperger and Eyrich, 2009a). The artery is easily damaged, such as through acute inflammation, and subsequent vascular disruption is critical in the establishment of mandibular OM. In addition, the canal acts as a corridor for the rapid dissemination of pus through the mandibular body, which further compresses the neurovascular tissues contained within it (Baltensperger and Eyrich, 2009a). Thus, for Moyoro 165, the inferior alveolar artery and mandibular canal may have contributed to and exacerbated the infection. Other than bone loss and evidence of dental tissue necrosis, no other characteristic signs of $\mathrm{OM}$ are evident on the mandible. However, given the young age of this individual, the unique manifestation of OM in the jaws, and the chronic nature of the condition, this is not unexpected (Aufderheide and Rodríguez-Martín, 1998; Ortner, 2003; Baltensperger and Eyrich, 2009a; Lewis, 2018; Roberts, 2019).

Chronic OM can persist for months or years. In the jaws, clinical symptoms such as localized swelling, pain, and/or numbness are usually less pronounced than those of the acute phase of the disease (Oulis et al., 2000; Belli et al., 2002; Baltensperger and Eyrich, 2009a; Chang et al., 2015; Fukuda et al., 2017). In some cases, patients can have no distress and even retain normal function such as mastication. The lytic alveolar lesion, mandibular canal destruction, and dental tissue loss on Moyoro 165 suggest that the infection was not clinically silent and had at least some impact on jaw function, if only temporary. However, the long-term survival of the child for months or even years after the onset of infection, and stable carbon and nitrogen isotope values consistent with other Moyoro individuals (Tsutaya et al., 2015: ID 1072 is Moyoro 165), imply adequate nutrition and care by family and community members even if masticatory function was impaired in some way. Chronic OM may have contributed to the death of the child, but other conditions cannot be ruled out as potential causes or contributing factors, especially considering the generally poor state of skeletal preservation. Indeed, the two linear enamel hypoplastic defects noted on the permanent mandibular canines (Figure S1) indicate physiological stress of some sort within the preceding 12-18 months.

While OM is considered to have been a relatively common condition in the past, especially among non-adult individuals (Baltensperger and Eyrich, 2009b; Santos and Suby, 2015; Roberts, 2019), it is rarely reported in the paleopathological literature (Tavares et al., 2017; Lewis, 2018; Roberts, 2019). Tavares and colleagues (2017) identified only ten published cases of pediatric OM, and a handful of others (e.g., Anderson and Carter, 1995; Ortner, 2003; Waters-Rist, 2012; Lewis, 2018) can be added to this list. OM of the jaws, in particular, has been documented even less frequently from archaeological human remains. Despite several reported infections being possibly attributable to jaw OM and/or odontogenic sources (Wood-Jones, 1910; Hooton, 1930; Roney, 1966; Khudaverdyan, 2011), we have identified only two other paleopathological cases involving non-adult individuals. In the first, Ortner (2003: 197-198) identified possible hematogenous spread of OM from a periapical maxillary abscess to the postcranial skeleton in a six-year-old child from 16th century AD Virginia (USA). In the second, Waters-Rist (2012) documented mandibular OM, likely stemming from an infected deciduous canine germ, in a 9to 18 -month-old infant from Early Neolithic ( 7000 cal. BP) Siberia. Lesion appearance in the latter case was considerably different from that presented here: there was evidence of suppuration and new periosteal bone formation, but no osteolytic defects or tooth loss. Thus, Moyoro 165 represents a unique manifestation of mandibular OM and, to the best of our knowledge, only the second pediatric example reported from an archaeological context. 


\section{Conclusion}

Moyoro 165 presents an unusual mandibular lesion with two distinct features, periosteal new bone formation and lytic alveolar destruction, that are most consistent with a diagnosis of OM. Direct extension from an odontogenic source, likely an infected tooth germ, is implicated by the lesion's location and its alveolar and dental involvement. Further lesion features, such as lamellated periosteal bone deposition and partial remodeling, suggest a chronic stage of infection over a period of months or even years that was still active at the time of death. Dental evidence of physiological stress during the previous $12-18$ months is consistent with this interpretation. Of the fewer than 20 cases of pediatric OM reported in the paleopathological literature, Moyoro 165 represents only the second involving the mandible, exhibiting features considerably different from those observed in the other case. The unique lesion appearance documented here highlights the diverse manifestation of OM, especially in the jaws and in the absence of modern therapeutic treatment. More research on OM during the pre-antibiotic eraincluding its variable prevalence, presentation, and distribution - is essential to more fully understand the condition's evolution and long-term impact on human populations.

\section{Funding}

This research was supported in part by the following: (1) Social Sciences and Humanities Research Council of Canada (Major Collaborative Research Initiative 412-2011-1001, Holocene Hunter-gatherers of Northeast Asia); (2) Japanese Society for the Promotion of Science Core to Core Program (Advanced Core Research Centre for the History of Human Ecology in the North); (3) MEXT KAKENHI (Grant-in-Aid for Scientific Research on Innovative Areas, 16H06408, Cultural History of Paleoasia)

\section{References}

Abashiri City Board of Education (2014) Shiseki Moyoro kaizuka seibijigyo houkokusho. Abashiri City Board of Education, Abashiri (in Japanese).

Akgül H.M., Çaglayan F., Yilmaz S.G., and Derindag G. (2018) Garre's osteomyelitis of the mandible caused by infected tooth. Case Reports in Dentistry, 1-4. DOI: 10.1155/2018/ 1409539

AlQahtani S.K., Hector M.P., and Liversidge H.M. (2010) The London atlas of human tooth development and eruption. American Journal of Physical Anthropology, 142: 481-490.

Amano T. (2003) Okhotsk-bunka toha nanika. In: Nomura T. and Utagawa Y. (eds.), Zoku-Jomon, Okhotsk bunka. Hokkaido Shimbun, Sapporo, pp. 110-133 (in Japanese).

Amberg S. and Ghormley R.K. (1934) Osteomyelitis among children. Journal of Pediatrics, 5: 177-193.

Anderson T. and Carter A.R. (1995) An unusual osteitic reaction in a young medieval child. International Journal of Osteoarchaeology, 5: 192-195, DOI: 10.1002/oa.139005214

Aufderheide A.C. and Rodríguez-Martín C. (1998) The Cambridge Encyclopedia of Human Paleopathology. Cambridge University Press, Cambridge.

Baltensperger M. and Eyrich G. (2009a) Osteomyelitis of the jaws: definition and classification. In: Baltensperger M. and Eyrich G. (eds.), Osteomyelitis of the Jaws. Springer, Berlin, pp.
5-56. DOI: 10.1007/978-3-540-28766-7_2

Baltensperger M. and Eyrich G. (2009b) Introduction. In: Baltensperger M. and Eyrich G. (eds.), Osteomyelitis of the Jaws. Springer, Berlin, pp. 1-3. DOI: 10.1007/978-3-540-2876672

Bancroft F.W. (1921) Acute hematogenous osteomyelitis. Annals of Surgery, 73: 681-700.

Baranoff A. (1934) The incidence of osteomyelitis of jaw bones among Chinese. Chinese Medical Journal, 48: 638.

Bass M.H. (1928) Acute osteomyelitits of the superior maxilla in young infants. American Journal of Diseases of Children, 35: $65-70$.

Belli E., Matteini C., and Andreano T. (2002) Sclerosing osteomyelitis of Garré periostitis ossificans. Journal of Craniofacial Surgery, 13: 765-768.

Binkley N. and Kreuger D. (2000) Hypervitaminosis A and bone. Nutrition Reviews, 58: 138-144.

Botía González C.M., Sirvent Cerdá S.I., and Azorín D. (2018) Bone metastasis of primitive occult neuroblastoma. Eurorad, DOI: 10.1594/EURORAD/CASE.15699

Caffey J. (1945) Pediatric X-Ray Diagnosis. Year Book Medical Publishers, Chicago.

Campanacci M., Bertoni F., and Bacchini P. (1990) Bone metastases from neuroblastoma. In: Campanacci M., Bertoni F., and Bacchini P. (eds.), Bone and Soft Tissue Tumors. Springer, Berlin, pp. 701-706.

Can I.H., Kurt A., Özer E., Sarì N., and Samim E. (2005) Mandibular manifestation of Langerhans cell histiocytosis in children. Oral Oncology Extra, 41: 174-177, DOI: 10.1016/j.ooe.2005. 04.007

Castelli W. (1963) Vascular architecture of the human adult mandible. Journal of Dental Research, 43: 786-792.

Chang Y.-C., Shieh Y.-S., Lee S.-P., Hsia Y.-J., Lin C.-K., Nieh S., Sytwu H.-K., and Chen Y.-W. (2015) Chronic osteomyelitis with proliferative periostitis in the lower jaw. Journal of Dental Sciences, 10: 450-455, DOI: 10.1016/j.jds.2012.09.020

Craig E. and Craig G. (2013) The diagnosis and context of a facial deformity from an Anglo-Saxon cemetery at Spofforth, North Yorkshire. International Journal of Osteoarchaeology, 23: 631-639, DOI: 10.1002/oa.1288

Dormans J.P. and Drummond D.S. (1994) Pediatric hematogenous osteomyelitis: new trends in presentation, diagnosis, and treatment. Journal of the American Academy of Orthopaedic Surgeons, 2: 333-341, DOI: 10.5435/00124635-19941100000005

Fédération Dentaire Internationale (FDI) (1971) Two-digit system of designating teeth. International Dental Journal, 21: 104106.

Fukuda M., Inoue K., and Sakashita H. (2017) Periostitis ossificans arising in the mandibular bone of a young patient: report of an unusual case and review of literature. Journal of Oral and Maxillofacial Surgery, 75: 1834.e1-1834.e8, DOI: 10.1016.j. jmos.2017.04.015

Grauer A.L. (2019) Circulatory, reticuloendothelial, and hematopoietic disorders. In: Buikstra J.E. (ed.), Ortner's Identification of Pathological Conditions in Human Skeletal Remains. Academic Press, London, pp. 491-529.

Hooton E. (1930) The Indians of Pecos Pueblo: A Study of their Skeletal Remains. Yale University Press, New Haven, CT.

Huang G.T.J. (2009) Endodontic infections in incompletely developed teeth. In: Fouad A.F. (ed.), Endodontic Microbiology. Wiley Blackwell, Ames, IA, pp. 261-280.

Hudson M.J. (2004) The perverse realities of change: world system incorporation and the Okhotsk culture of Hokkaido. Journal of Anthropological Archaeology, 23: 290-308, DOI: 10.1016/j. jaa.2004.05.002

Hwang D., Lee S., Kim U., Park H.R., Ryu M.H., Lee J.H., Jung Y., and Kim G.C. (2019) Langerhans cell histiocytosis of the mandible: two case reports and literature review. Journal of the Korean Association of Oral and Maxillofacial Surgeons, 
45: 167-172, DOI: 10.5125/jkaoms. 2019.45.3.167

Ishida H. (1988) Morphological studies of Okhotsk crania from Omisaki, Hokkaido. Journal of the Anthropological Society of Nippon, 96: 17-45.

Junno A., Dury J.P., Leipe C., Wagner M., Tarasov P.E., Hirasawa Y., Jordan P.D., and Kato H. (2021) Building a high-resolution chronology for northern Hokkaido-a case study of the Late Holocene Hamanaka 2 site on Rebun Island, Hokkaido (Japan). Journal of Archaeological Science: Reports, 36, 102867, DOI: 10.1016/j.jasrep.2021.102867

Kambadakone A.R., Kadavigere R.V., Hosahalli R.R., and Bhat S.S. (2008) Case report: CT features of cherubism. Indian Journal of Radiologic Imaging, 18: 56-59, DOI: 10.4103/09713026.38506

Kamoun-Goldrat A. and le Merrer M. (2008) Infantile cortical hyperostosis (Caffey disease): a review. Journal of Oral and Maxillofacial Surgery, 66: 2145-2150, DOI: 10.1016/j.joms. 2007.09.007

Karjodkar F.R. (2009) Textbook of Dental and Maxillofacial Radiology, 2nd edn. Jaypee, Panama City.

Khudaverdyan A.Y. (2011) The anthropology of infectious disease of Bronze Age and Early Iron Age from Armenia. Dental Anthropology, 24: 42-54.

Kiyono K. (1969) Nihon kaizuka no kenkyu. Iwanami shoten, Tokyo (in Japanese).

Kobayashi D., Satsuma S., Kamegaya M., Haga N., Shimomura S., Fujii T., and Yoshiya S. (2005) Musculoskeletal conditions of acute leukemia and malignant lymphoma in children. Journal of Pediatric Orthopaedics B, 14: 156-161, DOI: 10.1097/ 01202412-200505000-00003

Kodama S. (1948) Moyoro kaizuka. Hokkaido Genshi Bunka Kenkyukai, Sapporo (in Japanese).

Komai K. (1964) Okhotsk-kai engan Shiretoko-hanto no iseki. University of Tokyo Faculty of Letters, Tokyo (in Japanese).

Kondo O. and Aono T. (2016) Expansion of vertebral tuberculosis into Hokkaido: implications for the population history of Ainu. Quaternary International, 405(B): 58-65, DOI: 10.1016/j.quaint.2015.04.022

Kuzmin Y.V., Burr G.S., and Jull A.J.T. (2001) Radiocarbon reservoir correction ages in the Peter the Great Gulf, Sea of Japan, and eastern coast of the Kunashir, Southern Kurils (northwestern Pacific). Radiocarbon, 43: 477-481.

Lacey N.E. and Engle L.P. (1939) Acute osteomyelitis of the superior maxilla in children. Report of a case. Archives of Otolaryngology, 29: 417-427.

Lewis M. (2018) Paleopathology of Children. Identification of Pathological Conditions in the Human Skeletal Remains of Non-Adults. Academic Press, London.

Liepe C., Sergusheva E.A., Müller A., Spengler R.B., Goslar T., Kato H., Wagner M., Weber A.W., and Tarasov P.E. (2017) Barley (Hordeum vulgare) in the Okhotsk culture (5th-10th century $\mathrm{AD}$ ) of northern Japan and the role of cultivated plants in hunter-gatherer economies. PLoS ONE, 12: e0174397, DOI: 10.1371 .journal/pone/0174397

Marques C. (2019) Tumors of bone. In: Buikstra J.E. (ed.), Ortner's Identification of Pathological Conditions in Human Skeletal Remains. Academic Press, London, pp. 639-717.

Mitomi T., Tomizawa M., and Noda T. (2005) Tooth development included in the multifocal jaw lesions of Langerhans cell histiocytosis. International Journal of Paediatric Dentistry, 15: 123-126, DOI: 10.1111/j.1365-263X.2005.00603.X

Naito Y.I., Chikaraishi Y., Ohkouchi N., Mukai H., Shibata Y., Honch N.V., Dodo Y., Ishida H., Amano T., Ono H., and Yoneda M. (2010) Dietary reconstruction of the Okhotsk culture of Hokkaido, Japan, based on nitrogen composition of amino acids: implications for correction of ${ }^{14} \mathrm{C}$ marine reservoir effect on human bones. Radiocarbon, 52: 671-681.

Natvig P. and Dingman R.O. (1957) Osteomyelitis of the jaws in infants. American Journal of Surgery, 94: 873-876, DOI: 10.1016/0002-9610(57)90073-9
Naz J.F. and Edwards W.M. (1952) Hypervitaminosis A-a case report. New England Journal of Medicine, 246: 87-89, DOI: 10.1056/NEJM195201172460303.

Ortner D.J. (2003) Identification of Pathological Conditions in Human Skeletal Remains, 2nd edn. Academic Press, New York.

Oulis C., Berdousis E., Vadiakas G., and Goumenos G. (2000) Garre's osteomyelitis of an unusual origin in an 8-year-old child. A case report. International Journal of Paediatric Dentistry, 10: 240-244.

Papadaki M.A., Lietman S.A., Levine M.A., Olsen B.R., Kaban L.B., and Reichenberger E.J. (2012) Cherubism: best clinical practice. Orphanet Journal of Rare Diseases, 7(Suppl 1): S6, DOI: $10.1186 / 1750-1172-7-S 1-S 6$

Roberts C.A. (2019) Infectious disease: introduction, periostosis, periostitis, osteomyelitis, and septic arthritis. In: Buikstra J.E. (ed.), Ortner's Identification of Pathological Conditions in Human Skeletal Remains. Academic Press, London, pp. 285319.

Roberts C.A. and Buikstra J.E. (2019) Bacterial infections. In: Buikstra J.E. (ed.), Ortner's Identification of Pathological Conditions in Human Skeletal Remains. Academic Press, London, pp. 321-439.

Roksandic M. (2003) New Standardised Visual Forms for Recording the Presence of Human Skeletal Elements in Archaeological and Forensic Contexts. Internet Archaeology 13.

Roney J. (1966) Paleoepidemiology: an example from California. In: Jarcho S. (ed.), Human Paleopathology. Yale University Press, New Haven, CT, pp. 99-107.

Santos A.L. and Suby J.A. (2015) Skeletal and surgical evidence for acute osteomyelitis in non-adult individuals. International Journal of Osteoarchaeology, 25: 110-118, DOI: 10.1002/ oa. 2276

Sato T., Amano T., Ono H., Ishida H., Kodera H., Matsumura H., Yoneda M., and Masuda R. (2007) Origin and genetic feature of the Okhotsk people, revealed by ancient mitochondrial DNA analysis. Journal of Human Genetics, 52: 618-627, DOI: $10.1007 / \mathrm{s} 10038-007-0164-\mathrm{z}$

Sato T., Amano T., Ono H., Ishida H., Kodera H., Matsumura H., Yoneda M., and Masuda R. (2009) Mitochondrial DNA haplogrouping of the Okhotsk people based on analysis of ancient DNA: an intermediate of gene flow from the continental Sakhalin people to the Ainu. Anthropological Science, 117: 171-180, DOI: 10.1537 /ase. 081202

Schaefer M., Black S., and Scheuer L. (2009) Juvenile Osteology: A Laboratory and Field Manual. Academic Press, New York.

Suei Y., Taguchi A., and Tanimoto K. (2005) Diagnosis and classification of mandibular osteomyelitis. Oral Surgery, Oral Medicine, Oral Pathology, Oral Radiology, and Endodontology, 100: 207-214, DOI: 10.1016/j.tripleo.2004.11.006

Sugihara Y., Wakasa T., Kameyama T., Komatsu H., Kishi K., Koyama S., Nishijima Y., and Nishijima K. (1989) Pediatric acute lymphocytic leukemia with osseous changes in jaws: Literature review and report of a case. Oral Radiology, 5: 2531, DOI: 10.1007/BF02347843

Tavares A., Makhoul C., Monteiro M., and Curate F. (2017) Pediatric chronic osteomyelitis in the outskirts of Al-Ushbuna (Carnide, Lisboa, Portugal). International Journal of Paleopathology, 18: 1-4, DOI: 10.1016/j.ijpp.2017.06.003

Tsutaya T., Naito Y.I., Ishida H., and Yoneda M. (2014) Carbon and nitrogen isotope analyses of human and dog diet in the Okhotsk culture: perspectives from the Moyoro site, Japan. Anthropological Science, 122: 89-99, DOI: 10.1537/ase. 140604

Tsutaya T., Ishida H., and Yoneda M. (2015) Weaning age in an expanding population: stable carbon and nitrogen isotope analysis of infant feeding practise in the Okhotsk culture (5th-13th centuries) in Northern Japan. American Journal of Physical Anthropology, 157: 544-555, DOI: 10.1002/ajpa. 22740 
Ubelaker D.H. (1979) Human Skeletal Remains: Excavation, Analysis, and Interpretation. Smithsonian Institute Press, Washington, DC.

Walker A., Zimmerman M.R., and Leakey R.E.F. (1982) A possible case of hypervitaminosis A in Homo erectus. Nature, 296: 248-250, DOI: $10.1038 / 296248 \mathrm{a} 0$

Waters-Rist A.L. (2012) A unique case of mandibular osteomyelitis arising from tooth germ infection in a 7000-year-old infant from Siberia. Dental Anthropology, 25: 15-25, DOI: 10.26575/ daj.v25i1.55

Watzke I.M., Millesi W., Kermer C., and Gisslinger H. (2000) Multifocal eosinophilic granuloma of the jaw: long term follow-up of a novel interosseous corticoid treatment for recalcitrant lesions. Oral Surgery, Oral Medicine, Oral Pathology, Oral Radiology, and Endodontology, 90: 317-322, DOI: 10.1067. moe. 2000.107535

White S.C. and Pharoah M.J. (2009) Oral Radiology: Principles and Interpretation, 6th edn. Mosby, St Louis, MO.

Willman C.L., Busque L., Griffith B.B., Favara B.E., McClain K.L., Duncan H.M., and Gilliland D.G. (1994) Langerhans'-cell histiocytosis (histiocytosis X) - a clonal proliferative disease. New England Journal of Medicine, 331: 154160.

Willmon R., Coqueugniot H., Holowka S., Dutour O., and Pfeiffer S. (2013) Fibrous dysplasia of a temporal bone: a case from the Glen Williams ossuary, Ontario, Canada. International Journal of Paleopathology, 3: 269-273, DOI: 10.1016/j. ijpp.2013.09.006

Woodard W.K., Miller L.J., and Legant O. (1961) Acute and chronic hypervitaminosis in a 4-month-old child. Journal of Pediatrics, 59: 260-264, DOI: 10.1016/S0022-3476(61)80090-5

Wood-Jones F. (1910) General pathology (including diseases of the teeth). In: Elliot-Smith G. and Wood-Jones F. (eds.), The Archaeological Survey of Nubia Report for 1907-1908, Vol. II: Report on the Human Remains. National Printing Department, Cairo, pp. 263-292.

Yamaura K. (1998) The sea mammal hunting cultures of the Okhotsk Sea with special reference to Hokkaido prehistory. Arctic Anthropology, 35: 321-334.

Yamaura K. and Ushiro H. (1999) Prehistoric Hokkaido and Ainu origins. In: Fitzhugh W.W. and Dubreuil C.O. (eds.), Ainu: Spirit of Northern People. National Museum of Natural History, Smithsonian Institution, Washington, DC, pp. 39-46.

Yoneda M., Uno H., Shibata Y., Suzuki R., Kumamoto Y., Yoshida K., Sasaki A., and Kawahata H. (2007) Radiocarbon marine reservoir ages in the western Pacific estimated by pre-bomb molluscan shells. Nuclear Instruments and Methods in Physics Research B, 259: 432-437, DOI: 10.1016/j.nimb. 2007.01.184

Yu Q., Wang P.-Z., Shi H.-M., Wang L., and Sun D.-X. (1995) Radiographic findings in Langerhans' cell disease affecting the mandible. Oral Surgery, Oral Medicine, Oral Pathology, and Oral Radiology, 79: 251-254. 